\title{
Investigation of the feasibility to use Zeeman-effect background correction for the graphite furnace determination of phosphorus using high-resolution continuum source atomic absorption spectrometry as a diagnostic tool
}

\author{
Fábio G. Lepri ${ }^{\mathrm{a}}$, Bernhard Welz ${ }^{\mathrm{a}, *}$, Morgana B. Dessuy ${ }^{\mathrm{b}}$, Maria Goreti R. Vale ${ }^{\mathrm{b}}$, Denise Bohrer ${ }^{\mathrm{c}}$, \\ Margaretha T.C. de Loos-Vollebregt ${ }^{\text {d,e }}$, Mao Dong Huang ${ }^{f}$, Helmut Becker-Ross ${ }^{f}$ \\ a Departamento de Química, Universidade Federal de Santa Catarina, 88040-900 Florianópolis, SC, Brazil \\ b Instituto de Química, Universidade Federal do Rio Grande do Sul, Av. Bento Gonçalves, 9500, 91501-970 Porto Alegre-RS, Brazil \\ c Departamento de Química, Universidade Federal de Santa Maria, 97110-905 Santa Maria, RS, Brazil \\ d Delft University of Technology, Faculty of Applied Sciences, Analytical Biotechnology, Julianalaan 137, 2628 BL Delft, The Netherlands \\ e Department of Analytical Chemistry, Ghent University, Krijgslaan 281 - S12, B-9000 Ghent, Belgium \\ ${ }^{\mathrm{f}}$ ISAS, Institute for Analytical Sciences, Department of Interface Spectroscopy, Albert-Einstein Str. 9, 12489 Berlin, Germany
}

\section{A R T I C L E I N F O}

\section{Article history:}

Received 7 September 2009

Accepted 24 October 2009

Available online 6 November 2009

\section{Keywords:}

Phosphorus determination

Zeeman-effect background correction

Chemical modifiers

PO molecular absorption

High-resolution continuum source atomic

absorption spectrometry

\begin{abstract}
A B S T R A C T
The determination of phosphorus by graphite furnace atomic absorption spectrometry at the non-resonance line at $213.6 \mathrm{~nm}$, and the capability of Zeeman-effect background correction (Z-BC) to deal with the finestructured background absorption due to the $\mathrm{PO}$ molecule have been investigated in the presence of selected chemical modifiers. Two line source atomic absorption spectrometers, one with a longitudinally heated and the other with a transversely heated graphite tube atomizer have been used in this study, as well as two prototype high-resolution continuum source atomic absorption spectrometers, one of which had a longitudinally arranged magnet at the furnace. It has been found that Z-BC is capable correcting very well the background caused by the PO molecule, and also that of the NO molecule, which has been encountered when the Pd + Ca mixed modifier was used. Both spectra exhibited some Zeeman splitting, which, however, did not cause any artifacts or correction errors. The practical significance of this study is to confirm that accurate results can be obtained for the determination of phosphorus using Z-BC.

The best sensitivity with a characteristic mass of $m_{0}=11 \mathrm{ng} P$ has been obtained with the pure Pd modifier, which also caused the lowest background level. The characteristic mass obtained with the mixed $\mathrm{Pd}+\mathrm{Ca}$ modifier depended on the equipment used and was between $m_{0}=9 \mathrm{ng} \mathrm{P}$ and $m_{0}=15 \mathrm{ng} \mathrm{P}$, and the background signal was higher. The major problem of Z-BC remains the relatively restricted linear working range.
\end{abstract}

(C) 2009 Elsevier B.V. All rights reserved.

\section{Introduction}

Zeeman-effect background correction (Z-BC) was investigated thoroughly by de Loos-Vollebregt and de Galan [1] for its applicability in atomic absorption spectrometry (AAS), particularly with graphite tube atomizers. After its commercial introduction in the early 1980s [2] $\mathrm{Z}$-BC was considered the most efficient technique to correct for all kinds of background absorption that appears in graphite furnace AAS (GF AAS) [3]. In the meantime further advances have been achieved [4], and the technique is still considered the best choice for background correction in line source GF AAS (LS GF AAS). Nevertheless, when analyzing samples with high phosphorus content, some authors [5-8] reported errors that were related to the Zeeman splitting of the phosphorus monoxide (PO)

\footnotetext{
* Corresponding author. Fax: + 554837216850 .

E-mail address: w.bernardo@terra.com.br (B. Welz).
}

molecular spectrum under the influence of the magnetic field. Wibetoe and Langmyhr [5] found over- and under-correction errors for the determination of Pd in phosphoric acid at $247.6 \mathrm{~nm}$ and $244.8 \mathrm{~nm}$, respectively. These palladium atomic lines are situated close to PO absorption rotational lines as shown by Massmann et al. [6] using a highresolution spectrometer. Wibetoe and Langmyhr [5] also reported overcorrection for the determination of $\mathrm{Cu}$, Fe and In in the presence of phosphates. Zong et al. [7] found overcorrection for the determination of $\mathrm{Pb}$ using the absorption lines at $217.0 \mathrm{~nm}$ and $283.3 \mathrm{~nm}$, and Manning and Slavin [8] reported overcorrection for Ag determination using the 328.1-nm line. Heitmann et al. [9], using a prototype high-resolution continuum source GF AAS (HR-CS GF AAS) with inverse Z-BC, showed that the structured background absorption spectra in the vicinity of the 228.802-nm Cd line, attributed to PO, changed under the magnetic field used for Z-BC, resulting also in overcorrection.

Lepri et al. [10] investigated the determination of phosphorus using HR-CS GF AAS at the $213.618 \mathrm{~nm}$ non-resonance line in the 
absence and in the presence of selected chemical modifiers. Without a modifier the diatomic molecule PO was formed almost exclusively early in the atomization stage, when a phosphate, such as $\mathrm{NH}_{4} \mathrm{H}_{2} \mathrm{PO}_{4}$ was used to prepare the standard solutions. Independent confirmation of the formation of the PO molecule in a graphite furnace has been obtained by Sturgeon and Willie [11] using furnace atomization plasma emission spectrometry (FAPES). The molecular absorption spectrum is part of the $\mathrm{X}^{2} \Pi \rightarrow \mathrm{D}^{2} \Pi$ electronic transition of the PO molecule that exhibits a pronounced rotational fine structure [12]. In their studies of phosphorus atomization using HR-CS GF AAS Dessuy et al. [13] observed that a 'tail' appeared after the first sharp molecular absorption peak, which was shown to be due to atomic phosphorus. The proposed mechanism of atomization is a slow release of phosphorus atoms from the graphite tube wall following the adsorption of PO molecules at active sites of the graphite surface and their subsequent reduction to the element [13].

When phosphorus is measured with LS GF AAS at the non-resonance doublet at $213.5 / 213.6 \mathrm{~nm}$ without the addition of a modifier the absorption is to a significant percentage due to the PO molecule, the rotational 'lines' of which are overlapping in part with the radiation emitted by the line source. The extent to which the individual species contribute to the total absorbance depends very much on the experimental and instrumental conditions. At an atomization temperature of $2400{ }^{\circ} \mathrm{C}$ only PO is formed, but the contribution of atomic P increases with increasing temperature, and reaches about $50 \%$ at $2700{ }^{\circ} \mathrm{C}[13,14]$. Nevertheless, these measurements have been made using HR-CS AAS, and pixel width and the number of pixels used for measurement have an influence on the measured ratio, as the half width of the molecular absorption 'line' is greater than that of the atomic line. Yet another unknown parameter is the contribution of the line at $213.547 \mathrm{~nm}$ in LS AAS, which is about 10 times weaker than the line at $213.681 \mathrm{~nm}$, and has not been investigated using HR-CS AAS.

Palladium, alone or with the addition of calcium or ascorbic acid, was found to be the only modifier to produce almost exclusively atomic phosphorus. In the presence of lanthanum or sodium fluoride a mixture of atomic phosphorus and PO was produced, depending on the atomization temperature [10].

Dessuy et al. [14] investigated artifacts created when using LS GF AAS with deuterium lamp background correction $\left(D_{2}-B C\right)$ for the determination of phosphorus, using HR-CS AAS as a diagnostic tool. The molecular absorption due to $\mathrm{PO}$, which exhibits pronounced fine structure, could not be corrected by the $\mathrm{D}_{2}$ - $\mathrm{BC}$ system, as expected, causing significant overcorrection. Several artifacts, including underand overcorrection were observed in the presence of $\mathrm{NaF}, \mathrm{La}$ and $\mathrm{Pd}+$ $\mathrm{Ca}$ as the modifiers; satisfactory results could be obtained only in the presence of the Pd modifier.

The purpose of the present work has been to evaluate the determination of phosphorus in the absence and presence of different modifiers by LS GF AAS with a Z-BC system, and to investigate to which extent Z-BC is capable correcting for fine-structured background absorption. The experimental conditions were chosen to produce different phosphorus species in the atomization stage, such as atomic phosphorus in the presence of $\mathrm{Pd}$, a mixture of $\mathrm{P}$ and $\mathrm{PO}$ in the absence of a modifier and in the presence of $\mathrm{NaF}$, and $\mathrm{P}$ and $\mathrm{NO}$ in the presence of the mixed $\mathrm{Pd}+\mathrm{Ca}$ modifier. Four significantly different instruments, three of which were equipped with Z-BC, have been used in this investigation in order to minimize overinterpretation of results arising from any single spectrometer. Details of these instruments are given in the Experimental part.

\section{Experimental}

\subsection{Instrumentation}

Four different atomic absorption spectrometers have been used in this study, two conventional instruments with a line radiation source and two prototype HR-CS GF AAS instruments. Three of the instruments were equipped with a magnet at the furnace, as will be described in detail in the following.

The first instrument was a Zeeman 5100 atomic absorption spectrometer (Perkin-Elmer, Norwalk CT, USA), similar to that described by Fernandez et al. [2], equipped with an HGA 600 longitudinally heated graphite tube furnace with a transverse magnetic field and AS-60 auto sampler. A phosphorus hollow cathode lamp (Perkin-Elmer, Norwalk CT, USA) was used with a current of $35 \mathrm{~mA}$ and a spectral band pass of $0.7 \mathrm{~nm}$ at $213.6 \mathrm{~nm}$. Pyrolytically coated graphite tubes ( $28 \mathrm{~mm}$ long, $6 \mathrm{~mm}$ i.d.) with platform were used throughout.

The second one was a ZEEnit 600 atomic absorption spectrometer (Analytik Jena, Jena, Germany), similar to that described by Gleisner et al. [4], equipped with a transversely heated graphite tube atomizer, a transverse magnetic field and a furnace auto sampler. A phosphorus hollow cathode lamp (NARVA, GLE, Berlin, Germany) was used with a current of $5 \mathrm{~mA}$ and a spectral band pass of $0.5 \mathrm{~nm}$ at $213.6 \mathrm{~nm}$. Pyrolytically coated graphite tubes ( $19 \mathrm{~mm}$ long, $6 \mathrm{~mm}$ i.d.) with integrated PIN platform (Analytik Jena Part No. 407-152.011) were used throughout.

The third one was a prototype HR-CS GF AAS setup built at ISAS Berlin, which has been described in detail by Heitmann et al. [9]. It is based on a Perkin-Elmer Model 4100ZL atomic absorption spectrometer, from which the entire optical compartment including detector and controls have been removed and replaced by a double monochromator with a resolution of $\lambda / \Delta \lambda \approx 110,000$ and a charge coupled device (CCD) array detector. The conventional transversely heated graphite tube atomizer with a longitudinal magnetic field, supplied with the Model 4100ZL, has been used throughout. Pyrolytically coated graphite tubes ( $17 \mathrm{~mm}$ long, $5 \mathrm{~mm}$ i.d.) with integrated platform were used and solutions have been injected using an AS-71 Autosampler (Perkin-Elmer).

The fourth instrument was another prototype HR-CS GF AAS, also built at ISAS Berlin, which is based on an AAS 6 Vario (Analytik Jena, Jena, Germany), which has been modified in a similar way as described above. Details of this equipment were described previously $[12,15]$. The transversely heated graphite tube atomizer is very similar to that of the ZEEnit 600 , but without a Zeeman magnet, facilitating direct comparisons between the two instruments. Pyrolytically coated graphite tubes (19 mm long, $6 \mathrm{~mm}$ i.d.) with PIN platform (Analytik Jena Part No. 407-A81.025) have been used throughout.

The graphite furnace temperature program used for all determinations is shown in Table 1. Further details of the temperature programs used for specific measurements are mentioned in the text or the figure captions. Argon with a purity of $99.996 \%$ from different suppliers has been used throughout. Integrated absorbance (peak area) has been used for signal evaluation exclusively. Further details about specific conditions for some of the experiments are given in the respective figure captions and in the text.

Table 1

Graphite furnace temperature program used for the determination of phosphorus; gas flow rate $0.3 \mathrm{~L} \mathrm{~min}^{-1}$ in Perkin-Elmer furnaces and $2.0 \mathrm{~L} \mathrm{~min}^{-1}$ in Analytik Jena furnaces; gas flow interrupted in the atomization stage.

\begin{tabular}{|c|c|c|c|c|}
\hline \multirow{2}{*}{$\begin{array}{l}\text { Program } \\
\text { stage }\end{array}$} & Temperature & Ramp time ${ }^{a}$ & Ramp $^{b}$ & Hold time \\
\hline & $/{ }^{\circ} \mathrm{C}$ & $/ \mathrm{s}$ & $\overline{{ }^{\circ} \mathrm{C} \mathrm{s}^{-1}}$ & $/ \mathrm{s}$ \\
\hline Drying & 90 & 10 & 5 & 10 \\
\hline Drying & 100 & 10 & 5 & 10 \\
\hline Drying & 120 & 20 & 5 & 15 \\
\hline Pyrolysis & Variable & 10 & 100 & 15 \\
\hline Atomization ${ }^{\mathrm{c}}$ & 2700 & 0 & 3000 & 5 \\
\hline Cleaning & 2700 & 1 & 0 & 5 \\
\hline
\end{tabular}

a For the Perkin-Elmer furnaces.

b For Analytik Jena furnaces.

Read in this stage. 


\subsection{Reagents}

As the experiments have been carried out in different laboratories by different operators, the reagents used were on occasions from different suppliers. Analytical grade reagents were used unless otherwise specified. Nitric acid (Carlo Erba, Milan, Italy, No. 4080150) was further purified by double sub-boiling distillation using quartz sub-boiling still (Kürner Analysentechnik, Rosenheim, Germany). Water was purified in a Milli-Q system (Millipore, Bedford, MA, USA), resulting in water with a resistivity of $18 \mathrm{M} \Omega \mathrm{cm}$.

A phosphorus standard solution containing $10.0 \mathrm{~g} \mathrm{~L}^{-1} \mathrm{P}$ was prepared by dissolving the appropriate amount of $\mathrm{NH}_{4} \mathrm{H}_{2} \mathrm{PO}_{4}, 99 \% \mathrm{~m} / \mathrm{m}$ (Merck, Darmstadt, Germany or J. T. Baker, Phillipsburg, N.J., USA) in water. The solution was not acidified; the working standards were prepared by serial dilutions of the stock solution with water. Ten or $20 \mu \mathrm{L}$ of solution was introduced into the graphite tube for measurement.

A $2.0 \mathrm{gL}^{-1} \mathrm{NaF}$ modifier solution was prepared by dissolving sodium fluoride, $99 \% \mathrm{~m} / \mathrm{m}$ (Riedel-de-Haen or Merck) in water; the solution was not acidified. A $10 \mu \mathrm{L}$ volume of the modifier solution was introduced into the graphite tube, corresponding to a mass of $20 \mu \mathrm{g} \mathrm{NaF}$ in the graphite tube.

A palladium modifier solution containing $10 \pm 0.2 \mathrm{~g} \mathrm{~L}^{-1} \mathrm{Pd}$ in $15 \%$ $\mathrm{HNO}_{3}$ (Merck) or $1000 \mathrm{mg} \mathrm{L}^{-1} \mathrm{Pd}$ in $2 \% \mathrm{HNO}_{3}$ (Merck) was used, and a mass of 10 or $20 \mu \mathrm{g}$ Pd was introduced into the graphite tube. The palladium and calcium mixed modifier was prepared by adding a palladium modifier solution containing $1000 \mathrm{mg} \mathrm{L}^{-1} \mathrm{Pd}$ in $2 \% \mathrm{HNO}_{3}$ (Merck) together with a $1000 \mathrm{mg} \mathrm{L}^{-1} \mathrm{Ca}$ in $2 \% \mathrm{HNO}_{3}$ solution (Merck) into the graphite furnace. A mass of $10 \mu \mathrm{g}$ or $20 \mu \mathrm{g}$ Pd and $5 \mu \mathrm{g}$ Ca in $2 \%$ $\mathrm{v} / \mathrm{v} \mathrm{HNO}_{3}$ has been introduced into the graphite tube as the modifier.

\section{Results and discussion}

Phosphorus is atomized and/or volatilized in molecular form in GF AAS, and the extent to which one or the other species is formed depends on the experimental conditions, and particularly the chemical modifier used. The formation of molecular phosphorus species, mostly the monoxide PO, could cause interferences in the determination of phosphorus as the analyte, but also in the determination of other analytes, as detailed in the Introduction. The extent to which Zeeman-effect background correction (Z-BC) is capable of correcting this structured 'background' (which is actually due to the analyte itself) depends on whether the background without and with magnetic field is the same or not, i.e., if the PO molecule exhibits Zeeman splitting. Perfect correction could be expected if the molecular spectrum is not influenced by the magnetic field, whereas $\mathrm{BC}$ errors might be observed if the spectrum changes within the spectral interval that corresponds to the width of the line emitted by the radiation source.

\subsection{Atomization of phosphorus in the absence of a modifier}

In order to investigate this situation for the determination of phosphorus in the absence of a modifier, measurements have been carried out with a prototype HR-CS GF AAS with a longitudinally arranged magnet at the transversely heated graphite tube atomizer (HR-CS Z-GF AAS). The time-integrated wavelength-resolved absorbance spectrum of $0.5 \mu \mathrm{g}$ as $\mathrm{NH}_{4} \mathrm{H}_{2} \mathrm{PO}_{4}$ without a magnetic field is shown in Fig. 1a, the same spectrum with a magnetic field of about $0.8-0.9 \mathrm{~T}$ is shown in Fig. $1 \mathrm{~b}$, and the difference between the two spectra in Fig. 1c. The position of the atomic absorption line of $\mathrm{P}$ at $213.618 \mathrm{~nm}$ is indicated by the dashed line; the second atomic absorption line of $\mathrm{P}$ at $213.547 \mathrm{~nm}$ is not considered here, as it is about an order of magnitude weaker. It is clearly visible that the atomic line overlaps in part with the wing of a PO molecular 'line'. Although the spectra in Fig. 1a and b are not exactly identical, the differences (Fig. 1c) are not very significant and do not exceed an integrated absorbance of $\pm 0.01 \mathrm{~s}$; this means that no major artifacts should be expected for Z-BC. There is also a phosphorus atomic absorption signal visible in Fig. 1c, which is due to the secondary atomization that appears as a tail after the PO molecular absorption. It is worth mentioning that all spectra recorded with HR-CS AAS are automatically corrected for any continuous background that might appear in the following figures using LS AAS.

The measurements with line source AAS (LS AAS) have been carried out with two different instruments, one with a transversely heated furnace (TH-GF AAS), and one with a longitudinally heated furnace (LH-GF AAS), both with the magnets in a transverse arrangement. The transient absorbance signals for $5 \mu \mathrm{g}$ P and $10 \mu \mathrm{g}$ $\mathrm{P}$ are shown in Fig. 2a and b for TH-GF AAS and in Fig. 2c and d for LHGF AAS, respectively. There are obvious similarities but also some differences between the signals obtained with the two atomizers, which could mostly be associated with the differences in the furnace design. The most important common feature is that apparently both Z-BC systems can cope with the structured background without

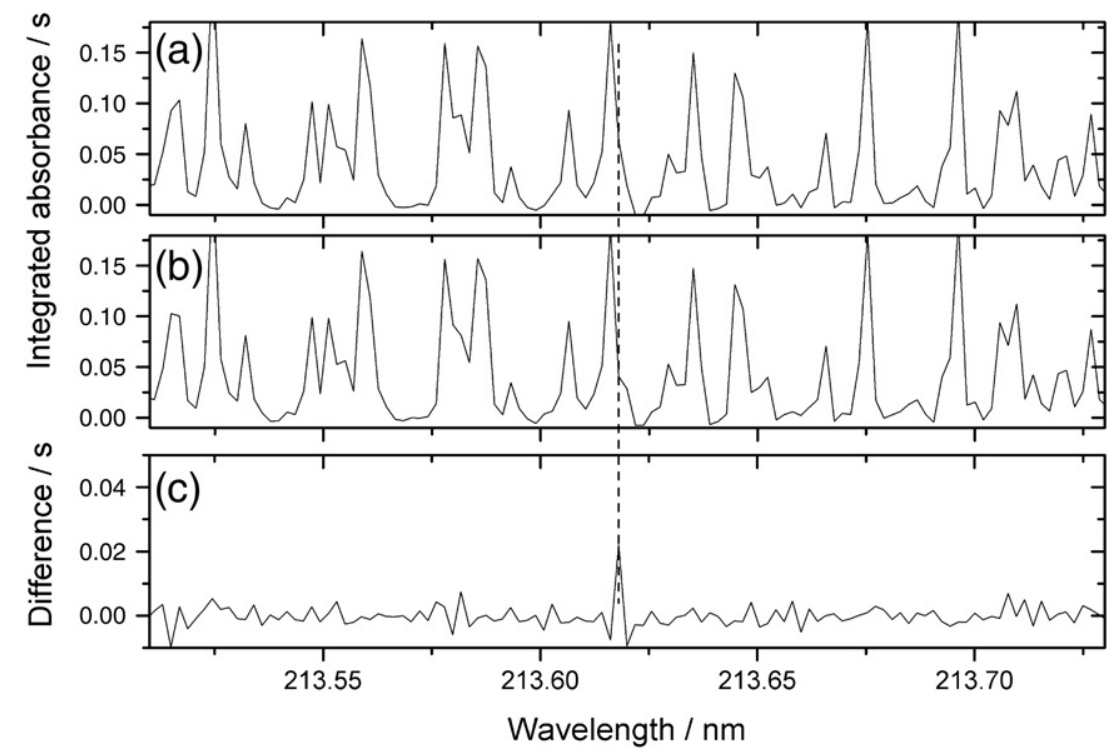

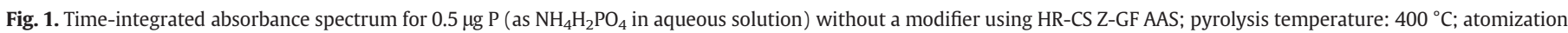

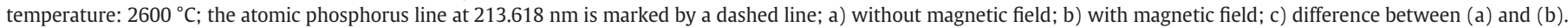



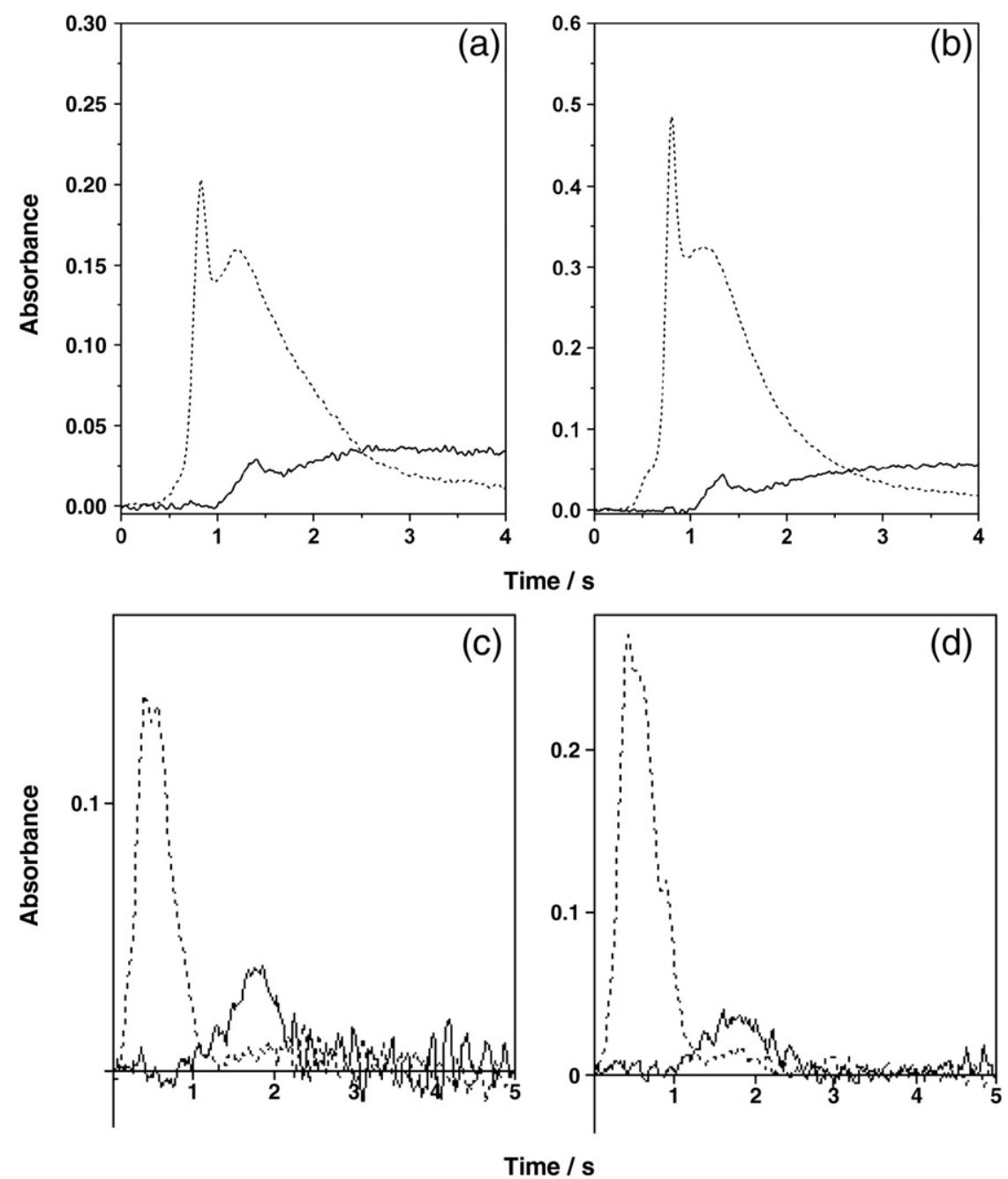

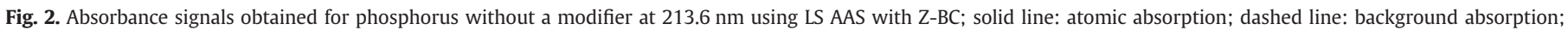
$T_{\text {pyr }}=400{ }^{\circ} \mathrm{C} ; T_{\text {at }}=2700^{\circ} \mathrm{C}$; a) and b) TH-GF AAS; c) and d) LH-GF AAS; a) and c) $5 \mu$ g P; b) and d) $10 \mu \mathrm{g} \mathrm{P}$.

problems and correct it to the baseline, i.e., no artifacts due to the molecular absorption can be observed.

One of the most obvious differences between the two furnaces is in the shape of the atomic $\mathrm{P}$ absorbance signal; whereas the signal extends over more than $3 \mathrm{~s}$ in the case of TH-GF AAS, a relatively sharp peak is obtained with LH-GF AAS. This phenomenon can be explained with the proposed mechanism of atomic $P$ formation [13]. In the case of TH-GF AAS we have a spatially isothermal atomizer, which means that the PO has much more opportunity to react with the hot graphite, and $\mathrm{P}$ atoms can be released for a much longer time from the tube surface. In the case of LH-GF AAS the ends of the graphite tube are up to $1500{ }^{\circ} \mathrm{C}$ cooler than the tube center [16], which promotes condensation of PO without re-atomization, except for the center of the graphite tube. The fact that the tailing is more pronounced in $\mathrm{LH}-$ GF AAS, compared to TH-GF AAS is supporting this assumption; another clear indication is the characteristic mass of $270 \mathrm{ng}$ found by TH-GF AAS, compared to $510 \mathrm{ng}$ found by LH-GF AAS. It is obvious that the $\mathrm{P}$ atomic absorption in Fig. $2 \mathrm{a}$ and $\mathrm{b}$ has not returned to the baseline, and that a longer integration time would have further improved the characteristic mass. This, however, has not been considered important in this context; the only important observation is that the contribution of atomic absorption to the total absorbance is much more pronounced in TH-GF AAS compared to LH-GF AAS.

The second difference between the two atomizers is the shape of the background absorption signal, which is quite sharp in the case of
LH-GF AAS, but exhibits a clear double peak with tailing in the case of TH-GF AAS. There is a small second background signal also visible in Fig. 2c and d, which, however, appears to be mostly due to the about $30 \%$ contribution of the $\sigma^{+/-}$components of the atomic P absorption. The strong second background absorption signal in Fig. 2a and b was also observed with HR-CS GF AAS [14]; it appeared to be without any fine structure, and was removed by the automatic background correction system of this equipment. This background was ascribed to another, yet unidentified $\mathrm{P}$ species, as only phosphorus was introduced into the graphite furnace. Interestingly, this background absorption in Fig. 2a and b was not corrected by $\mathrm{D}_{2}-\mathrm{BC}$ [14], and was actually recognized as atomic $\mathrm{P}$ and added to its absorbance, a phenomenon that has not yet been explained. As this second molecule is obviously less volatile than PO, it might be condensing and trapped at the cool tube ends very efficiently, so that it does not appear in LHGF AAS.

\subsection{The sodium fluoride modifier}

The stabilization mechanism of the NaF modifier for phosphorus is still not clear in spite of some preliminary theories given in the literature [17]. Anyway, it has been shown using HR-CS GF AAS that this modifier is capable of avoiding analyte losses up to a pyrolysis temperature of about $1000{ }^{\circ} \mathrm{C}$ [10], but that it cannot avoid the formation of a large quantity of PO, as has been confirmed with the HR-CS Z-GF AAS 


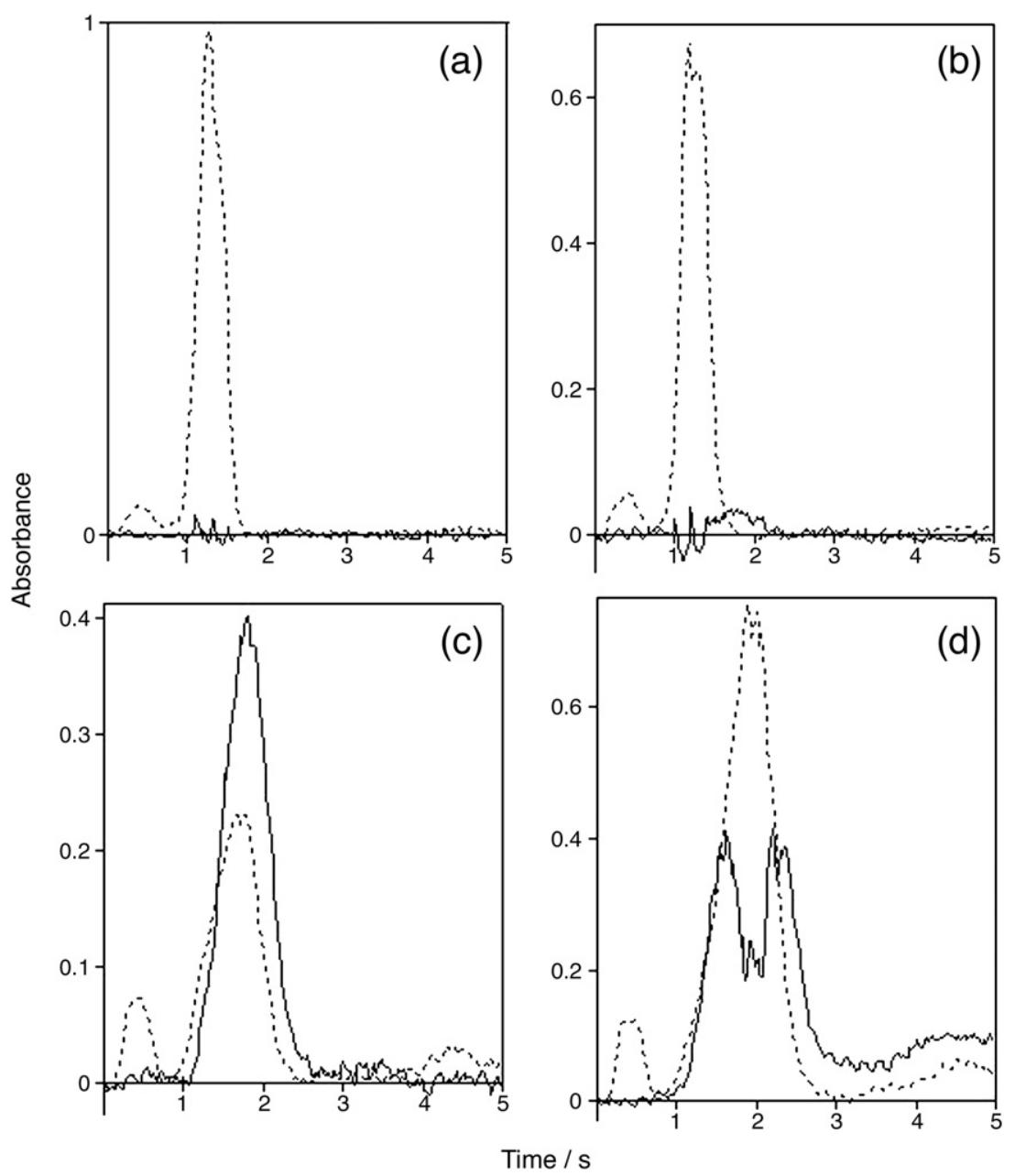

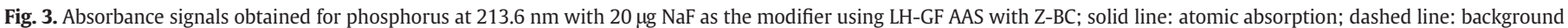
absorption; $T_{\mathrm{pyr}}=400{ }^{\circ} \mathrm{C} ; T_{\mathrm{at}}=2700{ }^{\circ} \mathrm{C}$; a) blank; b) $0.1 \mu \mathrm{g} \mathrm{P}$; c) $3.0 \mu \mathrm{g} \mathrm{P}$; d) $20 \mu \mathrm{g} \mathrm{P}$.

prototype. The spectrum was very similar to that in Fig. 1 and is not shown here; the only difference was that a significantly greater integrated absorbance signal for atomic phosphorus has been obtained in this case.

The NaF modifier shows some peculiarities that will be discussed in the following paragraphs. Firstly, the modifier itself produces a very high and sharp background signal early in the atomization stage, as is shown in Fig. 3a, which is corrected automatically in HR-CS GF AAS, as it is continuous within the recorded spectral interval. This background signal diminishes significantly already upon the addition of relatively low masses of $P$, as shown in Fig. 3b. Both figures also exhibit some baseline distortion due to the background absorption, which might be due to the very rapidly increasing absorbance signal. The background diminishes further for higher masses of $P$ although to a lesser extent, as shown in Fig. 3c, and it is increasing again when a very high mass of $\mathrm{P}$ is used, as shown in Fig. 3d. In the latter case it is obvious that the roll-over absorbance of about $A=0.4$ has been reached, and any increase of the analyte atom concentration in the absorption volume results in a reduction of the measured absorbance, exhibiting itself as a double peak [2].

The background absorption in the presence of the NaF modifier represents a relatively complex situation, as it actually consists of three sources of background: Firstly, there is the background absorption due to the NaF modifier, which is diminishing with increasing phosphorus mass, as has been observed previously [13]. It might be assumed that the phosphorus is 'consuming' part of the modifier that is causing the background absorption, although no chemical reaction can be offered for that at this point in time. Secondly, there is the PO absorption that is increasing with the analyte mass, and it is increasing more linearly, as it is much less affected by the magnetic field. Thirdly, there is the contribution of the $\sigma^{+/-}$ components, which is also increasing with the analyte content, and

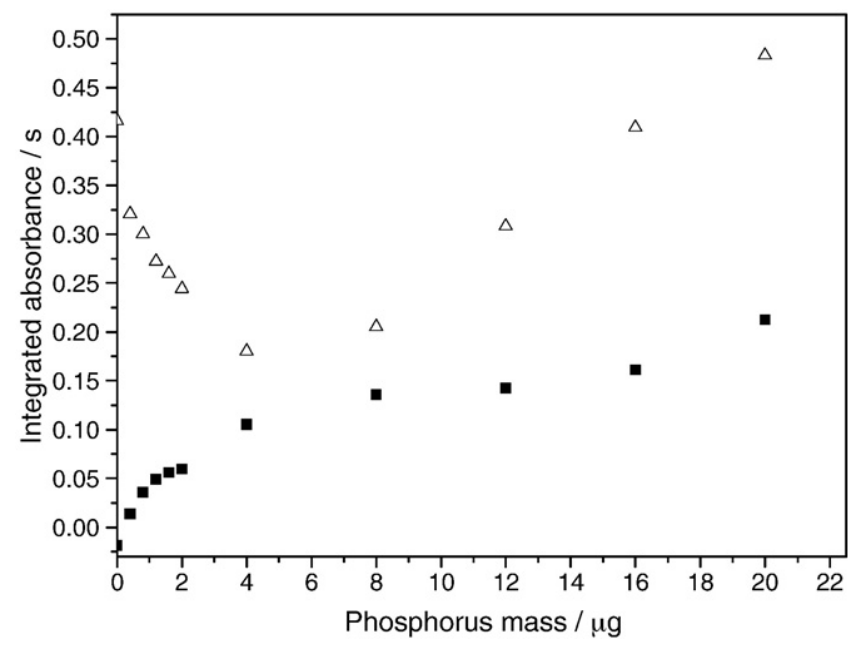

Fig. 4. Background-corrected calibration curve ( $\boldsymbol{\square})$ and background absorbance $(\Delta)$ measured for $\mathrm{P}$ in the presence of $20 \mu \mathrm{g} \mathrm{NaF}$ as the chemical modifier at $213.6 \mathrm{~nm}$ using TH-GF AAS with Z-BC. 

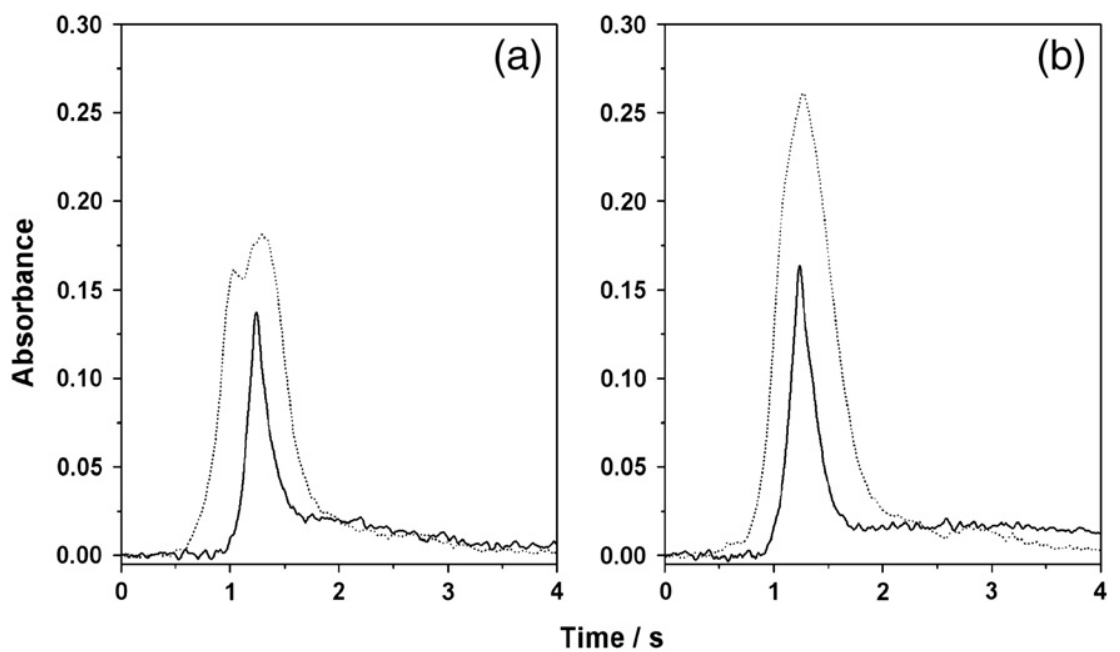

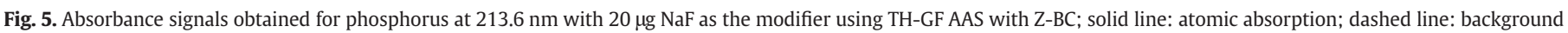
absorption; $T_{\text {pyr }}=1000^{\circ} \mathrm{C} ; T_{\text {at }}=2700{ }^{\circ} \mathrm{C}$; a) $1 \mu \mathrm{g}$ P; b) $2 \mu \mathrm{g} \mathrm{P}$.

also more linearly than the $\mathrm{P}$ atomic absorption. All these effects together are producing the phenomenon shown in Fig. 4 that the integrated atomic absorption is exhibiting pronounced non-linearity and is leveling off already at rather low integrated absorbance values; the background absorbance, in contrast, is first decreasing significantly with increasing analyte concentration, but then increasing again due to the contributions of the analyte to the background.

Although the overall behavior regarding analyte and background signals is very similar for LH-GF AAS and TH-GF AAS, there are also some distinct differences. Whereas the 'tail' due to the secondary atomization of $\mathrm{P}$ from $\mathrm{PO}$ adsorbed at the graphite tube wall becomes apparent in LH-GF AAS only for very high masses of P (refer to Fig. 3d), it appears for much lower P masses in TH-GF AAS as shown in Fig. 5a and $b$. The reason for that is the same as discussed in the previous section, i.e., a preferred condensation of $\mathrm{PO}$ at the cold tube ends of the LH-GF AAS that cannot support any secondary atomization of $\mathrm{P}$, which is in contrast to the isothermal conditions in the latter furnace. The late appearance of the tail in Fig. 3d compared to Fig. $2 a$ and b, and also $5 \mathrm{a}$ and $\mathrm{b}$ is apparently due to shift in the appearance of the PO absorption for high phosphorus mass in the presence of the modifier.

\subsection{The palladium modifier}

The use of Pd as a modifier allows using a pyrolysis temperature of $1600{ }^{\circ} \mathrm{C}$ for the determination of phosphorus. It has been observed previously that no molecular PO, but essentially only atomic P is formed in the presence of this modifier [10]. The efficiency of this modifier has already been reported by Sturgeon and Willie [11] using the FAPES source, as the emission of the PO molecule essentially disappeared in the presence of reduced palladium. This has been confirmed with measurements using the HR-CS Z-GF AAS prototype, as shown in Fig. 6. Without a magnetic field (Fig. 6a) only the two phosphorus absorption lines at $213.618 \mathrm{~nm}$ and $213.547 \mathrm{~nm}$ are visible, which broaden significantly when a magnetic field is applied, as shown in Fig. 6b. The difference in the phosphorus absorption with and without magnetic field is shown in Fig. 6 c. The ratio is 0.63 , which

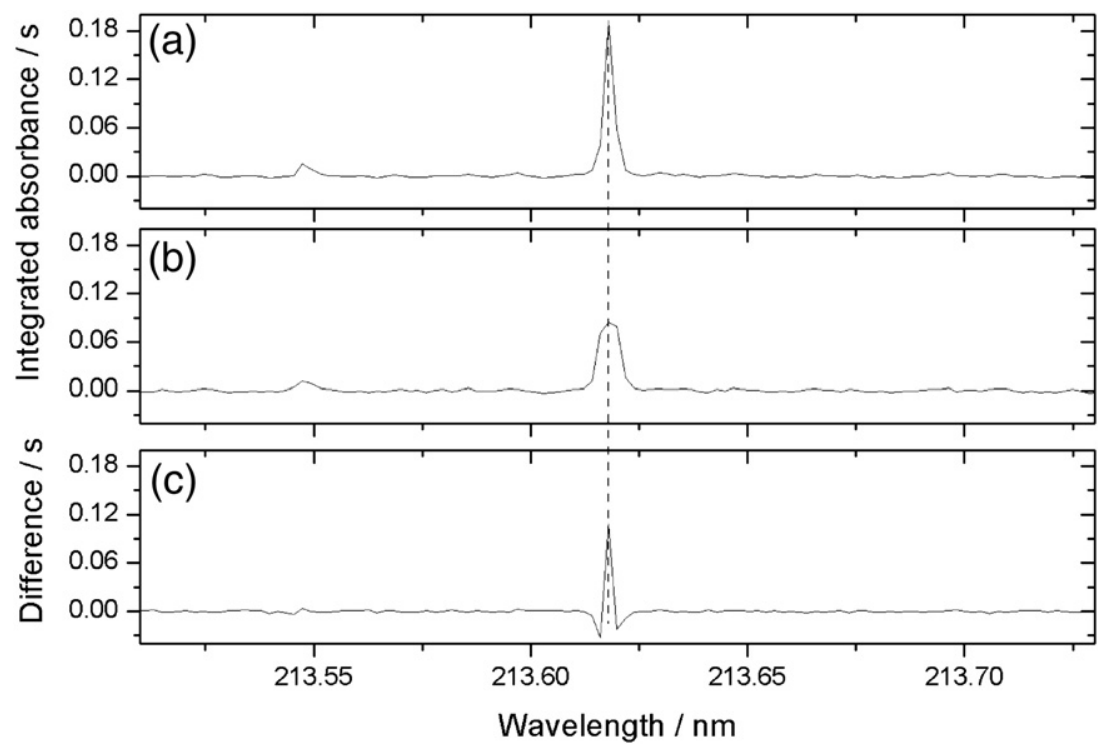

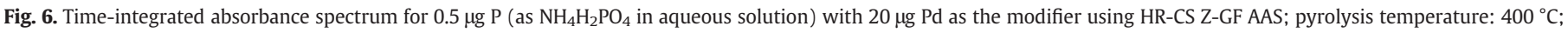

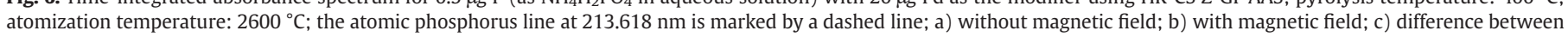
(a) and (b). 


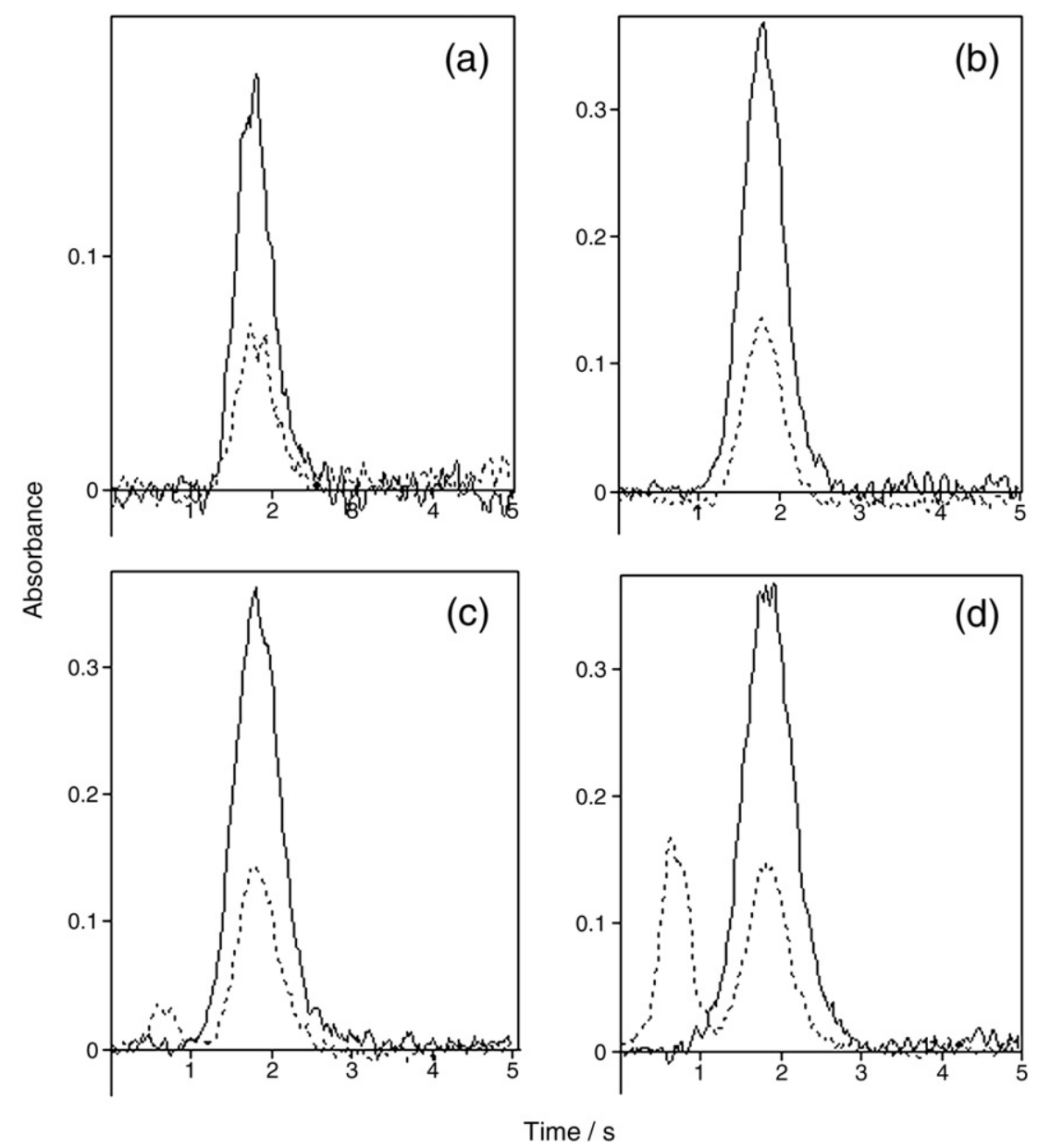

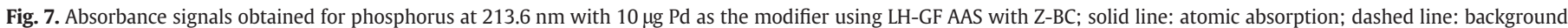
absorption; $T_{\mathrm{pyr}}=400{ }^{\circ} \mathrm{C} ; T_{\mathrm{at}}=2700{ }^{\circ} \mathrm{C}$; a) $0.2 \mu \mathrm{g} \mathrm{P}$; b) $2.0 \mu \mathrm{g} \mathrm{P}$; c) $7.0 \mu \mathrm{g}$ P; d) $20 \mu \mathrm{g} \mathrm{P}$.

is close enough to the theoretical value of 0.7 for a magnetic field of $0.9 \mathrm{~T}$ [18]. Obviously, when a line source is used, the emission profile is narrower than the absorption profile shown in Fig. 6a. The negative absorbance at both sides of the P absorption in Fig. $6 \mathrm{c}$ is an artifact of HR-CS GF AAS, which is due to the $\sigma$ components that are not overlapping with the emitted radiation in LS AAS, but become fully 'visible' in HR-CS AAS.

The absorbance signals obtained for analyte masses between $0.2 \mu \mathrm{g}$ and $20 \mu \mathrm{g}$ P using LH-GF AAS are shown in Fig. 7a-d. The background signal recorded for low phosphorus mass is typically around $35 \%$ of the integrated atomic absorbance signal, which corresponds to the Zeeman factor of 0.63 determined experimentally with the prototype HR-CS Z-GF AAS. This means that the background signal is entirely due to the overlap of the $\sigma^{+/-}$components of phosphorus with the line emitted by the radiation source. For analyte masses higher than $3 \mu \mathrm{g} \mathrm{P}$ and a modifier mass of $10 \mu \mathrm{g} \mathrm{Pd}$, an additional small background signal appeared early in the atomization stage, which increased significantly with the analyte mass as shown in Fig. 7c and $d$. There is no doubt that this background signal is due to $\mathrm{PO}$, and it is interesting to note that this signal appears when the molar ratio of 1 between the analyte and the modifier is exceeded. There is no tail visible in Fig. 7d although a considerable amount of PO is formed; however, comparing this signal to those in Fig. 2a and b, the tail might well appear together with the main $\mathrm{P}$ atomization signal, and hence not show up as a separate peak. Similar to the NaF modifier the phosphorus absorbance does not exceed an absorbance of about $A=0.4$, which appears to be the limiting absorbance for roll-over.

The calibration curve obtained with the palladium modifier using HR-CS AAS could be best described using two linear functions with significantly different slopes. This has been explained by two different stabilization mechanisms for phosphorus, one by palladium that is intercalated in the graphite structure and the other by palladium at the platform surface [14]. This behavior could not be observed in the present work using LS AAS with Z-BC, where the calibration curves exhibited a more 'conventional' shape, i.e., an increasing non-linearity over the entire calibration range, as shown in Fig. 8. This effect might

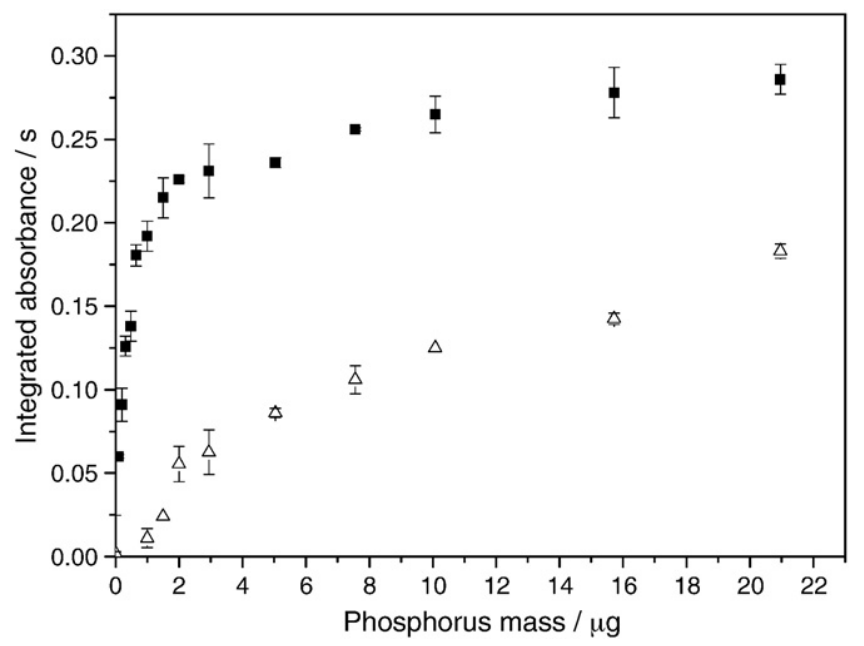

Fig. 8. Background-corrected calibration curve (-n-) and background absorbance $(-\Delta-)$ recorded at $213.6 \mathrm{~nm}$ with $10 \mu \mathrm{g}$ Pd as the modifier using LH-GF AAS with Z-BC; $T_{\text {pyr }}=400{ }^{\circ} \mathrm{C} ; T_{\text {at }}=2700^{\circ} \mathrm{C}$. 


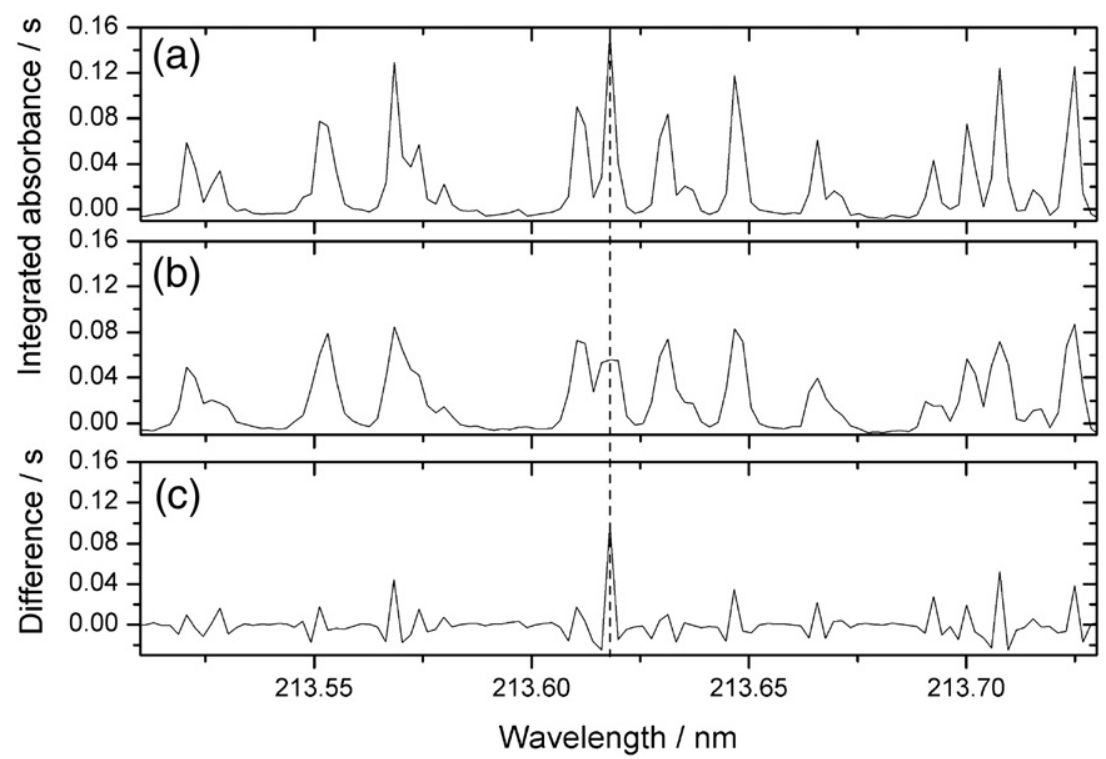

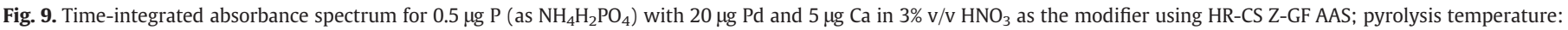

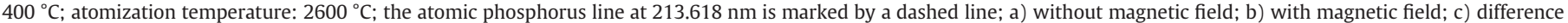
between (a) and (b).

actually have been masked by the well-known non-linearity of analytical curves obtained with Z-BC [1]. Results obtained with TH-GF AAS are not shown here, as they have not been significantly different from those with LH-GF AAS. The same characteristic mass of $m_{0}=11 \mathrm{ng}$ has been obtained with both instruments, although the tube of the TH-GF AAS is significantly shorter (19 mm versus $28 \mathrm{~mm}$ ), demonstrating the better efficiency in the latter one due to the isothermal atomization conditions.

\subsection{The palladium and calcium mixed modifier}

The palladium and calcium mixed modifier was recommended for the determination of $\mathrm{P}$ more than 20 years ago, giving the best sensitivity for this analyte [19]. However, it was also found more recently, using HR-CS GF AAS that this modifier is producing significant continuous and structured background, which is causing interference when $\mathrm{D}_{2}$-BC is used [14]. The structured background was found to be due to the NO molecule that is originating from the nitric acid content of the modifier, which is retained in the furnace up to pyrolysis temperatures of at least $1600{ }^{\circ} \mathrm{C}$ in the presence of calcium [10]. This situation is illustrated in Fig. 9a using the HR-CS Z-GF AAS system; the spectrum is part of the $\Delta v=+1$ sequence of the NO band system around $214 \mathrm{~nm}$, which is due to the electronic transition $\mathrm{X}^{2} \Pi \rightarrow \mathrm{A}^{2} \sum^{+}$ [12]. It is quite obvious from Fig. 9b and $\mathrm{c}$ that the NO molecular spectra without and with magnetic field are significantly different, i.e., exhibit pronounced Zeeman splitting.

In order to investigate to which extent this structured background absorption - which in this case is not due to the analyte, but to the modifier - could influence measurements using Z-BC, the determination of phosphorus has been investigated using LH-GF AAS and THGF AAS. The modifier blank and the signal for $0.5 \mu \mathrm{g} P$ obtained with the former equipment are shown in Fig. 10a and b. There is only small background absorption visible for the blank, which, however, does not cause any significant baseline distortion. Similarly, there are no anomalies visible in the absorption signal for $0.5 \mu \mathrm{g}$ P in Fig. 10b, and the background absorption is obviously due to the $\sigma^{+/-}$components of $P$ that are overlapping with line emitted by the radiation source.

\section{Conclusion}

It could be shown in this work that GF AAS with Zeeman-effect background correction is actually not suffering from any problems
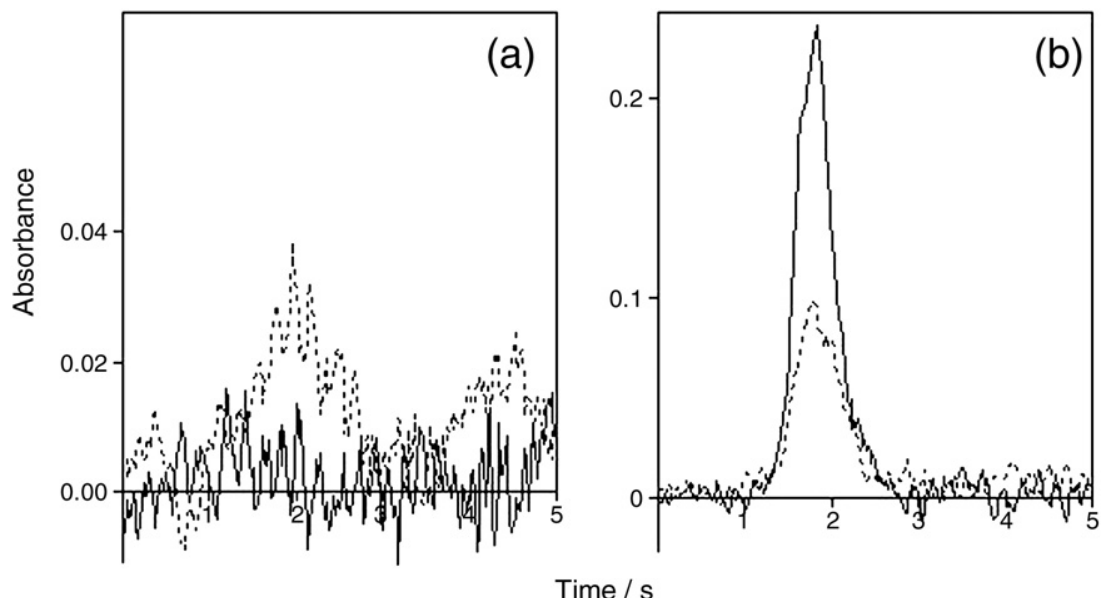

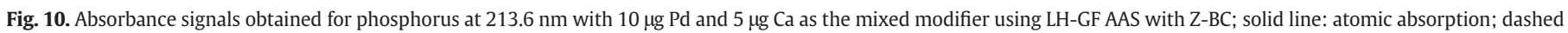
line: background absorption; $T_{\mathrm{pyr}}=400{ }^{\circ} \mathrm{C} ; T_{\mathrm{at}}=2700{ }^{\circ} \mathrm{C}$; a) modifier blank; b) $0.5 \mu \mathrm{g}$. 
with the fine-structured molecular absorption due to PO in the vicinity of the phosphorus non-resonance line at $213.618 \mathrm{~nm}$, although the PO rotational lines exhibit some moderate Zeeman splitting. The only modifier in the presence of which some baseline distortion has been observed for low phosphorus mass was the NaF modifier, which produced a very sharp and high background absorption early in the atomization stage. Even the mixed $\mathrm{Pd}+\mathrm{Ca}$ modifier did not cause any major baseline distortion, although it has been responsible for strong NO molecular absorption, which exhibited significant Zeeman splitting. Obviously, all this experience has been obtained in the vicinity of the phosphorus non-resonance line and for the determination of this analyte, and no extrapolation to other analytes and spectral ranges can be offered. This is particularly the case for the PO molecular absorption, which is relatively weak in the vicinity of the 213.618-nm phosphorus line compared to other spectral ranges [12].

\section{Acknowledgement}

The authors are grateful to Conselho Nacional de Desenvolvimento Científico e Tecnológico (CNPq) for financial support. F.G.L., B.W., M.B.D. and M.G.R.V. have research scholarships from CNPq. The authors are also grateful to Analytik Jena AG for the donation of the HR-CS AAS prototype.

\section{References}

[1] M.T.C. de Loos-Vollebregt, L. De Galan, Theory of Zeeman atomic absorption spectrometry, Spectrochim. Acta Part B 33 (1978) 495-511.

[2] F.J. Fernandez, W. Bohler, M.M. Beaty, W.B. Barnett, Atom. Spectrosc. 2 (1981) $73-80$.

[3] W. Slavin, D.C. Manning, G.R. Carnrick, The stabilized temperature platform furnace, Atom. Spectrosc. 2 (1981) 137-145.

[4] H. Gleisner, K. Eichardt, B. Welz, Optimization of analytical performance of a graphite furnace atomic absorption spectrometer with Zeeman-effect background correction using variable magnetic field strength, Spectrochim. Acta Part B 58 (2003) 1663-1678.

[5] G. Wibetoe, F.J. Langmyhr, Interference in inverse Zeeman-corrected atomic absorption spectrometry caused by Zeeman splitting of molecules, Anal. Chim. Acta 198 (1987) 81-86.
[6] H. Massmann, Z. El Gohary, S. Güçer, Analysenstörungen durch strukturierten Untergrund in der Atomabsorptionsspektrometrie, Spectrochim. Acta Part B 31 (1976) 399-409.

[7] Y.Y. Zong, P.J. Parsons, W. Slavin, Background correction errors for lead in the presence of phosphate with Zeeman graphite furnace atomic absorption spectrometry, Spectrochim. Acta Part B 53 (1998) 1031-1039.

[8] D.C. Manning, W. Slavin, Silver as a test element for Zeeman furnace AAS Spectrochim. Acta Part B 42 (1987) 755-763.

[9] U. Heitmann, M. Schütz, H. Becker-Ross, S. Florek, Measurements of Zeemansplitting of analytical lines by means of a continuum source graphite furnace atomic absorption spectrometer with a linear charge coupled device array, Spectrochim. Acta Part B 51 (1996) 1095-1105.

[10] F.G. Lepri, M.B. Dessuy, M.G.R. Vale, D.L.G. Borges, B. Welz, U. Heitmann, Investigation of chemical modifiers for phosphorus in a graphite furnace using high-resolution continuum source atomic absorption spectrometry, Spectrochim. Acta Part B 61 (2006) 934-944.

[11] R.E. Sturgeon, S.N. Willie, Excitation and detection of molecular species with furnace atomization plasma emission spectrometry, J. Anal. At. Spectrom. 7 (1992) 339-342.

[12] B. Welz, H. Becker-Ross, S. Florek, U. Heitmann, High-Resolution Continuum Source AAS, Wiley-VCH, Weinheim, Germany, 2005.

[13] M.B. Dessuy, M.G.R. Vale, F.G. Lepri, B. Welz, U. Heitmann, Investigation of phosphorus atomization using high-resolution continuum source electrothermal atomic absorption spectrometry, Spectrochim. Acta Part B 62 (2007) 429-434.

[14] M.B. Dessuy, M.G.R. Vale, F.G. Lepri, D.L.G. Borges, B. Welz, M.M. Silva, U. Heitmann, Investigation of artifacts caused by deuterium background correction in the determination of phosphorus by electrothermal atomization using highresolution continuum source atomic absorption spectrometry, Spectrochim. Acta Part B 63 (2008) 337-348.

[15] A.F. Silva, D.L.G. Borges, B. Welz, M.G.R. Vale, M.M. Silva, A. Klassen, U. Heitmann, Method development for the determination of thallium in coal using solid sampling graphite furnace atomic absorption spectrometry with continuum source, high-resolution monochromator and CCD array detector, Spectrochim. Acta Part B 59 (2004) 841-850.

[16] M. Sperling, B. Welz, J. Hertzberg, C. Rieck, G. Marowsky, Temporal and spatial temperature distributions in transversely heated graphite tube atomizers and their analytical characteristics for atomic absorption spectrometry, Spectrochim. Acta Part B 51 (1996) 897-930.

[17] E. Hernández, J. Alvarado, F. Arenas, M. Vélez, Thermal stabilization of phosphorus during electrothermal atomic absorption spectrometry using sodium fluoride as a chemical modifier, J. Anal. At. Spectrom. 12 (1997) 1391-1396.

[18] B. Welz, M. Sperling, Atomic Absorption Spectrometry, 3rd edn., Wiley-VCH, Weinheim, Germany, 1999, p. 127.

[19] A.J. Curtius, G. Schlemmer, B. Welz, Determination of phosphorus by graphite furnace atomic absorption spectrometry: Part 2. Comparison of different modifiers, J. Anal. At. Spectrom. 2 (1987) 115-124. 